\title{
Aspartame and Sucralose-induced Fatty Changes in Rat Liver
}

\author{
Nadia Haq1', Raafea Tafweez1', Sadia Saqib1, Zahra Haider Bokhari1, Irfan Ali2 and Ahmed Fawad Syami3 \\ IDepartment of Anatomy, King Edward Medical University, Lahore, Pakistan \\ ${ }^{2}$ Department of Physiotherapy, Mayo Hospital, Lahore, Pakistan \\ ${ }^{3}$ Department of General Surgery, Govt Mohammad Nawaz Sharif Hospital, Yakki Gate, Lahore, Pakistan
}

\begin{abstract}
Objective: To determine the cytoplasmic fatty change of rat liver due to artificial sweeteners - aspartame and sucralose. Study Design: Experimental study.

Place and Duration of Study: Department of Anatomy, King Edward Medical University, Lahore, in collaboration with the University of Veterinary and Animal Sciences Lahore, from May to October 2016.

Methodology: Adult Wistar albino rats were randomly divided into five groups. Group I was control group (animals were given drinking water by oral gavage). The animals of group II and III were given low (40 mg/kg body weight) and high dose (1000 mg/kg body weight) of aspartame, respectively. Animals of group IV and V were given low (5 mg/kg body weight) and high dose (1000 mg/kg body weight) of sucralose respectively by oral gavage. Doses were given once daily, six days a week for a total duration of 8 weeks. At the end of experiment, livers of all animal groups were observed for fatty change of cytoplasm.

Results: Artificial sweeteners cause cytoplasmic fatty change of varying degrees. This change was more marked in high dose group of aspartame (group III) as compared to low dose group (group II). In sucralose group, this change was seen only in high dose group (group V).

Conclusion: Both artificial sweeteners-induced fatty changes in rat liver; the effect was least pronounced with low dose of sucralose.
\end{abstract}

Key Words: Artificial sweeteners, Rat liver, Fatty change, Aspartame, Sucralose.

How to cite this article: Haq N, Tafweez R, Saqib S, Bokhari ZH, Ali I, Syami AF. Aspartame and sucralose-induced fatty changes in rat liver. J Coll Physicians Surg Pak 2019; 29(9):844-51.

\section{INTRODUCTION}

Added sugars have been defined by the FDA as "sugars that are added to food during preparation and processing" excluding sugars naturally present in foods. 1 The American Heart Association recently released a scientific statement recommending added sugar intake to no more than 100 to 150 kilocalories per day for most Americans. ${ }^{2}$

Sugars are replaced by artificial sweeteners for a number of conditions. ${ }^{3}$ Diabetes mellitus becomes easily manageable because the total amount of sugar intake is reduced.4 Sugar substitutes are effective in the treatment of reactive hypoglycemia because foods rich in such sugars do not lead to insulin release, leading to hypoglycemia. ${ }^{5}$ They also help in weight loss by reduction of added sugars in diet, ${ }^{6}$ and as not fermented by the micro flora of dental plaque so helpful for good dental health. ${ }^{7}$ They are less costly and have longer shelf-life than sugars. ${ }^{3}$

Artificial sweeteners are of nutritive and non-nutritive types. ${ }^{8}$ Non-nutritive sweeteners add no energy to food. ${ }^{6}$

Correspondence to: Dr. Nadia Haq, Department of Anatomy,

King Edward Medical University, Lahore, Pakistan

E-mail: haqnadiaa@gmail.com

Received: September 19, 2018; Revised: April 28, 2019;

Accepted: May 25, 2019
Five sweeteners have been approved by Food and Drug Association (FDA): ${ }^{9}$ acesulfame potassium, saccharin, neotame, aspartame and sucralose. Among them, aspartame and sucralose are the two most commonly used sweeteners.

In the United States, beverages containing alternative sweeteners were used by $66 \%$ population in 2003-2004, while eatables with nutritive sweeteners were used by $82.3 \%$ people. 8,10

Like other amino acids, aspartame completely breaks down in the body to its basic components phenylalanine $50 \%$, aspartic acid $40 \%$ and $10 \%$ methanol by enzymatic degradation. ${ }^{11}$ Methanol alcohol breaks down into formaldehyde, which is oxidised to formate in the liver and results in the formation of free oxygen radicals. If taken in excess, the metabolic machinery of liver fails to handle it, leading to serious health problems. ${ }^{11,12}$

The rat liver is multilobed and its fundamental structure is similar to the human liver. 13 Microscopically, each lobe of liver consists of hexagonal hepatic lobules (classical lobule) made up of $65-70 \%$ of parenchymal cells (hepatocytes) and $35-40 \%$ of non-parenchymal cells; endothelial cells, Kupffer cells, Ito cells and Pit cells. ${ }^{12}$ Periportal hepatocytes get maximally oxygenated blood being specialised for oxidative functions and are more affected at the time of oxidative stress as compared to paracentral (zone III) hepatocyte. ${ }^{14}$ 
The rationale of this study was to see the cytoplasmic fatty change induced by aspatrame and sucralose. In the light of results obtained from this study, information will also be available to formulate recommendations for choice between these two commonly used artificial sweeteners.

The aim of the study was to determine the effects of aspartame and sucralose on the liver of albino rats.

\section{METHODOLOGY}

Fifty adult Wistar albino rats of both gender (average age between 2 to 3 months) and weight between 175225 gms were randomly divided into five groups and kept in separate labelled cages including control and experimental groups. Low and high doses of aspartame and sucralose were administered to albino rats with oral gavage. Control group was given $3 \mathrm{ml}$ of distilled water at each time segment of experiment.

As the ADI (acceptable daily intake) of aspartame in humans is $40 \mathrm{mg} / \mathrm{kg}$ body weight, 15 so low dose for group II animals was calculated to be $8 \mathrm{mg}$ for an average $200 \mathrm{gm}$ weight rat and high dose for group III animals was calculated to be $200 \mathrm{mg}$ for an average 200 $\mathrm{gm}$ rat as LD50 of oral aspartame is $>10,000 \mathrm{mg} / \mathrm{kg}$ in rats. ${ }^{16} \mathrm{ADI}$ of sucralose is $5 \mathrm{mg} / \mathrm{kg}$ body weight in humans, 17 so low dose for group IV animals was calculated to be $1 \mathrm{mg}$ for an average $200 \mathrm{gm}$ weight rat and high dose for group $\mathrm{V}$ animals was calculated to be $200 \mathrm{mg}$ for an average $200 \mathrm{gm}$ rat as LD50 of oral sucralose is $>10 \mathrm{~g} / \mathrm{kg}$ body weight in rats. ${ }^{18}$

After 8 weeks of experiment, all animals in each group were anaesthetised and liver was removed following rat dissection protocol. ${ }^{19} \mathrm{After}$ tissue processing slides of rat liver were stained with haematoxylin and eosin. 20 The image on microscope was focused with $40 \mathrm{X}$ objective and eyepiece being 10X giving total magnification of 400X. The photomicrography was also done using camera Nikon DS-Ri2 attached with microscope Nikon ECLIPSE Ci. The cytoplasmic fatty change of hepatocytes was closely observed in all slides and description of cytoplasm change (fatty change).

Qualitative variables were expressed as frequencies and percentages for each group. Chi-square test was applied for comparison of this qualitative variable when required. Data was stored and analysed by using SPSS version 21.0 in which $p$-value less than 0.05 was taken as statistically significant.

\section{RESULTS}

In the control group, normal looking homogenous eosinophilic cytoplasm was seen in all hepatocytes with centrally placed normal looking nuclei with well-defined nuclear membranes (Figure 1A). In low dose group of aspartame, focal cytoplasmic changes in the form of micro-vesicular fatty change were seen in three animals of this group (Table I, Figure 1B). These changes were only limited in centrilobular zones. Cytoplasmic changes were quite obvious in high dose group of aspartame. In half animal slides of this group, normal homogenous cytoplasmic staining was lost and focal areas of cytoplasmic changes in the form of micro- and macro-

Table I: Group-wise comparison of cytoplasmic changes in experimental groups by using Chi-square test.

\begin{tabular}{l|l|c|c|l}
\hline Group (I) & Group (J) & $\begin{array}{c}\text { Present } \\
\mathrm{n} \%\end{array}$ & $\begin{array}{c}\text { Absent } \\
\mathrm{n} \%\end{array}$ & p-value \\
\hline Group - I & Group - II & 30 & 70 & $0.03^{*}$ \\
& Group - III & 50 & 50 & $0.003^{*}$ \\
& Group - IV & 0 & 100 & 0.00 \\
& Group - V & 10 & 90 & 0.23 \\
\hline Group - II & Group - III & 50 & 50 & 0.359 \\
& Group - IV & 0 & 100 & $0.03^{*}$ \\
& Group - V & 10 & 90 & 0.255 \\
\hline Group - III & Group - IV & 0 & 100 & $0.003^{*}$ \\
& Group - V & 10 & 90 & $0.044^{*}$ \\
\hline Group - IV & Group - V & 10 & 90 & 0.23 \\
\hline
\end{tabular}

Group -l: Control group

Group -II: Experimental group (Low dose of apartame).

Group -III: Experimental group (High dose of aspartame).

Group -IV: Experimental group (Low dose of sucralose).

Group -V: Experimental group (High dose of sucralose).

$n=$ No. of animals.

*Significant difference among groups.

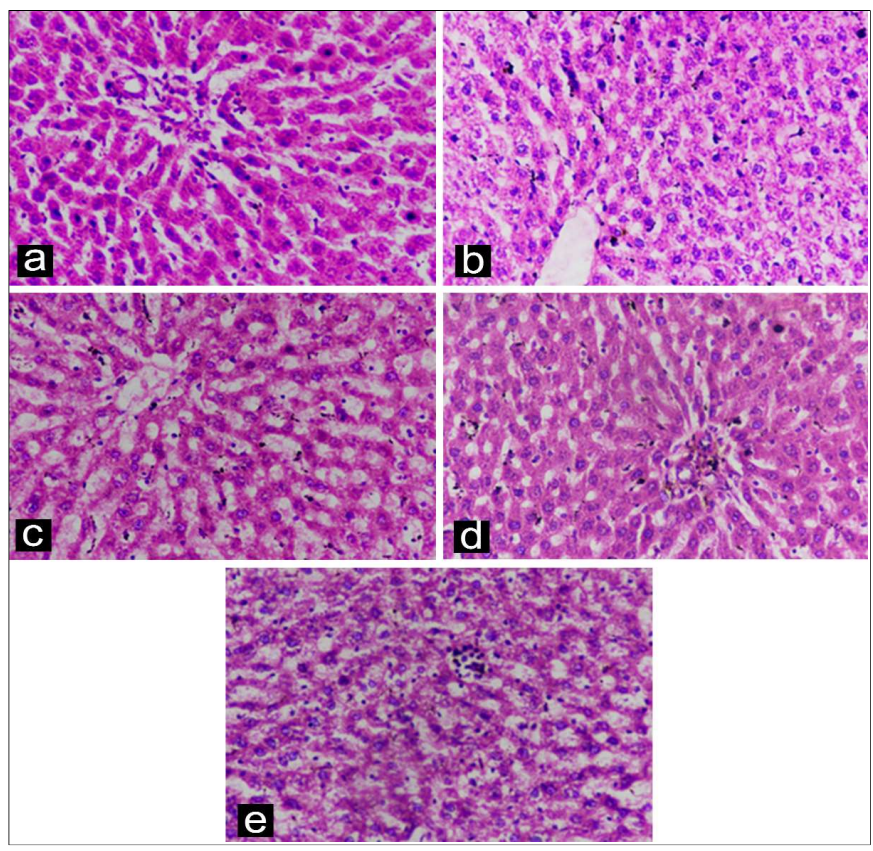

Figure 1: Cytoplasmic changes seen in various experimental groups on light microscope.

(A) Photomicrograph of control group (I). Normal looking cytoplasm and nucleus (H\&E).

(B) Photomicrograph of low dose group of aspartame (II). Enlarged hepatocytes with cytoplasmic fatty vacuolation (fatty change) are visible (H\&E).

(C) Photomicrograph of high dose group of aspartame (III) with moderate cytoplasmic vacuolation visible (H\&E) .

(D) Photomicrograph showing normal looking hepatocytes arranged in cords with sinusoids seen in low dose group of sucralose (IV). Cytoplasm is normal looking. Portal triad (portal vein, hepatic artery and bile ductule) is also visible (H\&E). Magnification $40 \mathrm{X} \times 10 \mathrm{X}=400 \mathrm{X}$.

(E) Photomicrograph showing hepatocytes arranged in cords with sinusoid are seen in high dose group of sucralose $(\mathrm{V})$. Enlarged hepatocytes with mild cytoplasmic fatty change were seen (H\&E).

Magnification $40 \mathrm{X} \times 10 \mathrm{X}=400 \mathrm{X}$. 
vesicular fatty change were seen in centrilobular as well as in periportal zones (Table I, Figure 1C).

Normal homogenous cytoplasmic staining remained preserved in low dose group of sucralose (Table I, Figure 1D). In high-dose group of sucralose microvesicular fatty change was seen in only one animal slide in periportal zone (Table I, Figure 1D).

Group-wise comparison showed that cytoplasmic changes of low and high dose groups of aspartame were statistically significant from control group. Animals treated with low and high doses of sucralose did not show statistically significant difference from control group (Table I).

\section{DISCUSSION}

A convincing sign of degenerative change in liver is cytoplasmic vacuolation or fatty change. In this study, fatty change was found both in low as well as high dose group of aspartame (Table I, Figures 1B and 1C). In lowdose group of aspartame cytoplasmic fatty change of micro-vesicular type was seen in centrilobular zones only; whereas, in high dose group of aspartame, same type of cytoplasmic fatty change was observed in centrilobular as well as in periportal zones. Among sucralose administered groups, cytoplasmic vacuolation in the form of micro-vesicular fatty change was only observed in one slide of high dose group in periportal zone (Table I, Figure 1E). Similar observations were made by Bothaina $M$. Khidr when aspartame was given equivalent to $200-1000 \mathrm{mg} / \mathrm{kg}$ body weight to two experimental groups; but the changes seen were more pronounced in high dose group. ${ }^{13}$

The fact that cytoplasmic changes are seen more in aspartame groups as compared to suralose groups can be explained on the basis of metabolic differences between these two sweeteners. Mourad explained the underlying cause of cytoplasmic fatty change to be a state of oxidative stress in tissues such as liver. 21 When aspartame is metabolised in liver cells, it leads to the formation of reactive metabolites which are normally handled by kidneys through enzymatic degradation. When consumed in large doses, these reactive metabolites can lead to the formation of reactive oxygen species (ROS). In later stage, balance of ROS production and its removal is disturbed leading to a state of oxidative stress, which is damaging to the tissues at cellular level. 22 These ROS react with macro-molecules such as proteins, lipids and DNA. ${ }^{22}$ Lipids are essential components of nuclear and cellular membranes. As mitochondria are energy houses of cells, after DNA damage fat which is normally processed for energy starts accumulating inside the cells and produce fatty change. During the process of pathogenesis, initially small fat vacuoles collect around the nucleus called micro-vesicular fatty change, later coalesce pushing nucleus to the periphery (signet ring) called macrovescular fatty change. 23

Alkafafy et al. also observed hepatocytes showing signs of degenerative changes in the form of vacuolar cytoplasm with aspartame in doses of $250 \mathrm{mg}$ to 1000 $\mathrm{mg} / \mathrm{kg}$.b.wt. given to albino rats. ${ }^{13}$

Sharma et al. documented cytoplasmic vacuolisation in liver of mice fed with high dose $(1000 \mathrm{mg})$ of sucralose. 24 Explanation given for fatty change induced by sucralose is different since minimal amount of sucralose is absorbed through gut. It is suggested by Jiang et al. that sucralose can cause liver damage by enhancing the growth of gut bacteria that are more efficient in getting energy from our food and turning that into stored fat leading to increased risk of liver diseases. These altered gut microbiota also impair the immune function of gut as a result colonic bacteria can have toxic effects on human host after intestinal absorption and transfer to the liver via portal vein which also causes hepatic steatosis and steatohepatitis. 25

The fatty change observed in the present study can be due to the oxidative stress; but confirmation of establishment of this fact remains beyond the scope of present study. Further studies should be carried out to explore the damaging effects of artificial sweeteners of other types and to see their effects after prolonged exposure. Effects on other vital organs and biochemical parameters should also be explored.

\section{CONCLUSION}

Aspartame was harmful to the liver even in low dose; whereas, sucralose is damaging to liver when taken in high dose. It is recommended that the facts about harmful effects of these artificial sweeteners should be brought to the notice of medical personnel as well as highlighted to the general population, specially the diabetics and the obese.

\section{CONFLICT OF INTEREST:}

Authors declared no conflict of interest.

\section{AUTHORS' CONTRIBUTION:}

$\mathrm{NH}$ : Planned the design of the work; analysed data for the work; Drafted the work ond revised it critically for important intellectual content; Approved the final version to be published; agreed to be accountable for all aspects of the work in ensuring that questions related to the accuracy or integrity of any part of the work are appropriately investigated and resolved.

RT: Planned the design of the work; analysed, or interpreted the data for the work; Drafted the work or revised it critically for important intellectual content.

SS: Planned the design of the work; analysed, or interpreted the data for the work; Drafted the work or revised it critically for important intellectual content; 
Approval the version to be published.

ZHB, IA, AFS: Drafted the work or revised it critically for important intellectual content.

\section{REFERENCES}

1. Erickson J, Slavin J. Total, added, and free sugars: Are restrictive guidelines science-based or achievable? Nutrients 2015; 7: 2866-78.

2. Eckel RH, Jakicic JM, Ard JD, De Jesus JM, Miller NH, Hubbard VS. Guideline on lifestyle management to reduce cardiovascular risk: A report of the American College of Cardiology/American Heart Association Task Force on Practice Guidelines. J Am Coll Cardiol 2014; 63:2960-84.

3. Geoffrey L. Glycemic responses and toleration. In: Q'Donnell K, Kearsley M, Eds. Sweeteners and sugar alternatives in food technology. 2nd ed. Cadbury UK. John Wiley \& Sons. 2012: p.1-26.

4. Hu FB. Resolved: There is sufficient scientific evidence that decreasing sugar-sweetened beverage consumption will reduce the prevalence of obesity and obesity-related diseases. Obes Rev 2013; 14:606-19.

5. Gonzalez A, Delgado M, Fraga-Fuentes MD. Use of diazoxide in management of severe postprandial hypoglycemia in patient after Roux-en-Y gastric bypass. Surg Obes Relat Dis 2013; 9:18-9.

6. Benton D. Can artificial sweeteners help control body weight and prevent obesity? Nutr Res Rev 2005; 18:63-76.

7. Ly KA, Milgrom P, Rothen M. Xylitol, sweeteners, and dental caries. Pediatr Dent 2006; 28:154-63.

8. Brown RJ, Banate MA, Rother KI. Artificial sweeteners: A systematic review of metabolic effects in youth. Pediatr Obes 2010; 5:305-12.

9. Hellsten I, Dawson J, Leydesdorff L. Implicit media frames: Automated analysis of public debate on artificial sweeteners. Public Underst Sci 2010; 19:590-608.

10. Brizuela $A B$, Raschi $A B$, Castillo $M V$, Leyton $P$, Romano $E$, Brandán SA. Theoretical structural and vibrational properties of the artificial sweetener sucralose. Comput Theor Chem 2013; 1:52-60.

11. Aspartame: By far the most dangerous substance added to most foods today [Updated on 2011, Cited on 2017]. Available at: http://articles.mercola.com.
12. Powell GM, Miller JJ, Olavesen AH, Curtis CG. Liver as major organ of phenol detoxication? Nature 1974; 252:234-5.

13. Alkafafy ME, Ibrahim ZS, Ahmed MM, El-Shazly SA. Impact of aspartame and saccharin on the rat liver: Biochemical, molecular, and histological approach. Int $J$ Immunopath Pharmacol 2015; 28:247-55.

14. Rappaport AM. The microcirculatory acinar concept of normal and pathological hepatic structure. Beitr Pathol 1976; 157: 215-43.

15. Renwick AG. Acceptable daily intake and the regulation of intense sweeteners. Food Addit Contam 1990; 7:463-75.

16. Abdel Messih NM. The safety of aspartame. USURJ 2015; 1:92-8.

17. Chattopadhyay S, Raychaudhuri U, Chakraborty R. Artificial sweeteners: A review. J Food Sci Technol 2014; 51:611-21.

18. Goldsmith LA. Acute and subchronic toxicity of sucralose. Food Chem Toxicol 2000; 38:S53-69.

19. Wingerd BD. Rat dissection manual. 1st ed. USA. JHU Press. John Hopkins University. 1988; 1:1-62.

20. Bancroft JD, Layton C. The hematoxylins and eosin. Bancroft's theory and practice of histological techniques. Elsevier 2013; 173-86.

21. Mourad M. Effect of aspartame on some oxidative stress parameters in liver and kidney of rats. Afr J Pharm Pharmaco 2011; 5:678-862.

22. Du Z, Yang Q, Liu L, Li S, Zhao J, Hu J, et al. NADPH oxidase 2-dependent oxidative stress, mitochondrial damage and apoptosis in the ventral cochlear nucleus of D-galactoseinduced aging rats. Neuroscience 2015; 286:281-92.

23. McPherson S, Hardy T, Henderson E, Burt AD, Day CP, Anstee QM. Evidence of NAFLD progression from steatosis to fibrosingsteatohepatitis using paired biopsies: Implications for prognosis and clinical management. J Hepatol 2015; 62:1148-55.

24. Sharma A, Panwar S, Singh AK, Jakhar KK. Studies on the genotoxic effects of sucralose in laboratory mice. Indian J Anim Res 2007; 41:1-8.

25. Jiang W, Wu N, Wang X, Chi Y, Zhang Y, Quu X, et al. Dysbiosis gut microbiota associated with inflammation and impaired mucosal immune function in intestine of humans with nonalcoholic fatty liver disease. Sci Rep 2015; 5:8096. 